\title{
ON SQUARE ROOTS OF NORMAL OPERATORS ${ }^{1}$
}

\author{
C. R. PUTNAM
}

1. All operators in this paper are bounded (linear, everywhere defined) transformations on a Hilbert space of elements $x$. An arbitrary operator $A$ will be called a square root of a normal operator $N$ if

$$
A^{2}=N \text {. }
$$

It is clear that if $N$ possesses the spectral resolution $N=\int z d K(z)$, then any operator of the form $A=\int z^{1 / 2} d K(z)$, where, for the value of $z^{1 / 2}$, the choice of the branch of the function may depend on $z$, is a solution of (1). Moreover, all such operators are even normal.

Of course, equation (1) may have other, nonnormal, solutions $A$. The object of this note is to point out a simple condition to be satisfied by a square root $A$ guaranteeing that it be normal. This criterion will involve the (closed, convex) set $W=W_{A}$ consisting of the closure of the set of values $(A x, x)$ where $\|x\|=1$. (Cf. also [2] wherein is discussed a connection between commutators and the set $W$.)

The following theorem will be proved:

(I) Let $N$ be a fixed normal operator and let $A$ denote an arbitrary solution of (1). Suppose that there exists a line $L$ in the complex plane passing through the origin and lying entirely on one side of (and possible lying all, or partly, in) the set $W_{A}$. Then $A$ is necessarily normal.

It is easy to see that the hypothesis of (I) concerning the line $L$ is surely satisfied if $W$ is a single point or a straight line segment. In this case, $A$ is even the sum of multiples of a self-adjoint operator and the unit operator $I$. (In fact, there exists some angle $\theta$ and some complex number $z$ such that the set $W$ belonging to $e^{i \theta} A+z I$ is a point or a segment of the real axis, and hence $e^{i \theta} A+z I$ is self-adjoint.) In case the set $W$ is actually two-dimensional, the assumption amounts to supposing that 0 is not in the interior of $W$, although it is allowed of course that 0 be on the boundary.

2. Proof of (I). Clearly, one can choose an angle $\theta$ for which the operator $B=e^{i \theta} A$ satisfies $B+B^{*} \geqq 0$. If $B=H+i J$, where $H$ $=\left(B+B^{*}\right) / 2$ and $J=-i\left(B-B^{*}\right) / 2$ denote the self-adjoint real and imaginary parts of $B$, then

Received by the editors October 10, 1956.

1 This research was supported in part by the United States Air Force under Contract No. AF 18(603)-139. 


$$
B^{2}=e^{2 i \theta} A^{2}=\left(H^{2}-J^{2}\right)+i(H J+J H) .
$$

Since $B^{2}$ is normal and obviously commutes with $B$, it follows that $B^{2}$ also commutes with $B^{*} ;[1]$. Consequently $B^{2}$ commutes with each of the operators $H$ and $J$. A subtraction of the two relations obtained from (2) by a multiplication by $H$ on the left and on the right, respectively, now implies $R+i S=0$, where $R=J^{2} H-H J^{2}$ and $S=H^{2} J-J H^{2}$. On taking adjoints, one obtains $R-i S=0$. Therefore $S=0$, that is $H^{2} J=J H^{2}$; hence, since $H \geqq 0, H J=J H$. Consequently $B$, hence $A$, is normal and the proof of (I) is now complete.

3. The following is a corollary of (I) and its proof:

(II) Let $N$ be a fixed self-adjoint operator and let $A$ denote a solution of (1) for which either (a) $\Re(A x, x) \neq 0$ or (b) $\Im(A x, x) \neq 0$ holds for all $x$. Then either $A$ or $i A$ is self-adjoint according as (a) or (b) holds.

It should be noted that the hypothesis of (II) implies that the line $L$ of (I) can be chosen either as the imaginary axis or as the real axis according as (a) or (b) holds and that, moreover, no number $(A x, x)$, for $\|x\|=1$, actually lies on $L$ (although, of course, such numbers may cluster at a point of $L$ ).

In order to prove (II), note that the angle $\theta$ occurring in the proof of (I) can now be chosen to be 0 or $\pi$ in case (a) and $\pi / 2$ or $3 \pi / 2$ in case (b). Furthermore, $(H x, x)>0$ whenever $\|x\|=1$, so that 0 is not in the point spectrum of $H$. Since $e^{2 i \theta}$ is real, it follows from the relation (2) that $H J+J H=0$. This fact combined with the relation $H J-J H=0$ implies $H J=0$, hence $J=0$. Thus $B(=H)$ is self-adjoint and so $A=e^{-i \theta} B$. In view of the choice of $\theta$, the proof of (II) is now complete.

\section{REFERENCES}

1. B. Fuglede, $A$ commutativity theorem for normal operators, Proc. Nat. Acad. Sci. U.S.A. vol. 36 (1950) pp. 35-40.

2. C. R. Putnam, On commutators and Jacobi matrices, Proc. Amer. Math. Soc. vol. 7 (1956) pp. 1026-1030.

Purdue University 